\title{
Neonatal Chest Wall Rhabdomyosarcoma
}

\author{
Michael Feldman $^{1} \quad$ Zvi Steiner $^{2}$ Gabriel Groisman ${ }^{3}$ Erez Nadir ${ }^{1}$ \\ ${ }^{1}$ Department of Neonatology, Hillel Yaffe Medical Center, \\ Hadera, Israel \\ 2 Department of Paediatric Surgery, Hillel Yaffe Medical Center, \\ Hadera, Israel \\ ${ }^{3}$ Department of Pathology, Hillel Yaffe Medical Center, Hadera, Israel
}

\begin{abstract}
Address for correspondence Erez Nadir, MD, Department of Neonatology, Hillel Yaffe Medical Center, Hadera 38100, Israel (e-mail: erezn@hy.health.gov.il).
\end{abstract}

Eur J Pediatr Surg Rep 2015;3:46-49.

\author{
Abstract \\ Keywords \\ - rhabdomyosarcoma \\ - newborn \\ - chest wall \\ - tumor
}

An infant was born at term with a huge chest mass diagnosed as rhabdomyosarcoma. Treatment consisted of surgical resection and chemotherapy. We describe this very rare congenital mass and the problematic therapeutic management of such a tumor in a newborn.

\section{Introduction}

Rhabdomyosarcoma is a malignant tumor of immature mesenchymal cell origin. It is the most common soft tissue sarcoma in children, but extremely rare among neonates. ${ }^{1}$ There are four recognized histological subtypes and diagnosis is performed by biopsy. Treatment options include surgical resection, chemotherapy, and radiotherapy, depending on the group of the tumor. We describe a case of neonatal rhabdomyosarcoma and the special dilemma it posed in therapeutic management.

\section{Case Report}

A male infant was born at term with a huge mass on his left chest. Apgar scores were 9 (at 1 minute) and 9 (at 5 minutes). Birth weight was $3,170 \mathrm{~g}$. The infant was transferred to the neonatal intensive care unit. The physical examination was normal except for a firm mass on the left chest ( $\sim$ Fig. 1 ). It measured $3.8 \times 2.8 \mathrm{~cm}$ on the sonographic study, on which it was seen to be well defined from the surroundings and located between the skin and the pectoralis major muscle, with a small central calcification and containing some peripheral blood vessels.

A trucut biopsy of the lesion ( - Fig. 2), performed on the 3rd day of life, showed fibrous and skeletal muscle infiltrated by sheets and bundles of eosinophilic spindle cells with dark,

received

August 11, 2014

accepted after revision

December 16, 2014

published online

January 28, 2015 round-to-oval nuclei displaying numerous mitoses and areas of coagulative necrosis. Immunohistochemical stains revealed that the tumor cells were diffusely positive for vimentin, focally positive for desmin and myogenin, and negative for CD-34, S-100 protein, ALK-1, actin, cytokeratin, and epithelial membrane antigen. The proliferation index with the $\mathrm{K}_{\mathrm{i}}-67$ marker was 40 to $50 \%$. The histopathologic differential diagnosis was between congenital or infantile fibrosarcoma and

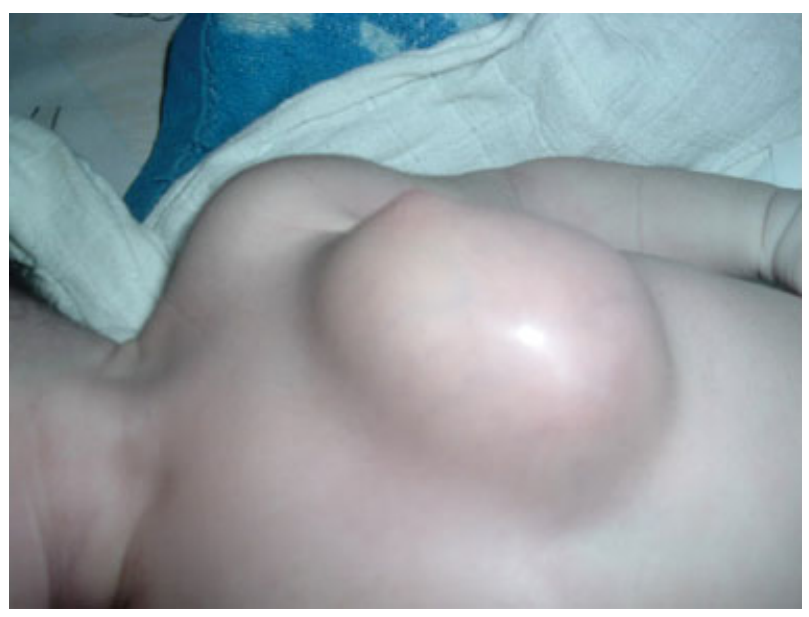

Fig. 1 Chest mass on the 1st day.
License terms Stuttgart · New York 


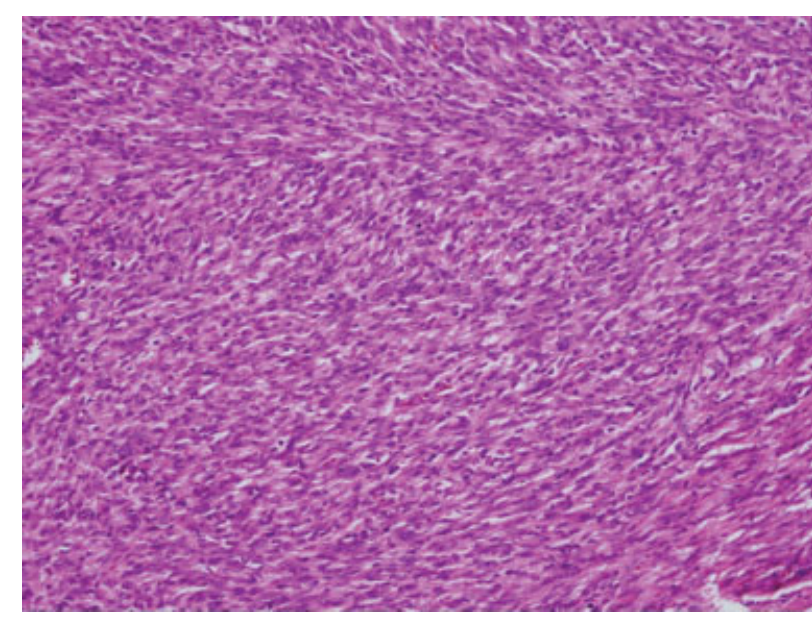

Fig. 2 Presumed diagnosis of fibrosarcoma with suspected local invasion.

embryonal rhabdomyosarcoma. The lesion was finally diagnosed an embryonal rhabdomyosarcoma by an international expert on pediatric soft tissue tumor cells.

Unfortunately, as there was no magnetic resonance imaging (MRI) device in the hospital in which the infant was born, and due to nonmedical considerations, chest MRI was performed in another medical center only when the infant was 13 days of age ( - Fig. 3). At that time the lesion was $3 \times 4.6 \times 6.7 \mathrm{~cm}$ and had nodular components. It involved the skin and subcutis tissue as well as the chest wall, including distortion and indentation of the left hemithorax. At the lower aspect, there was irregularity of the anterior chest wall and intercostal thickening was suspected. There was no evidence of flow void in the lesion. Breast tissue was developed symmetrically to the contralateral side and pushed anteriorly by the lesion. The lesion was consistent with fibrosarcoma, with suspected local invasion. No lymph node involvement and no metastases were demonstrated.

At 16 days of age, the size of the mass on the left chest had doubled since birth ( - Fig. 4). Due to fast growing up rate of the lesion, we decided to skip the step of neoadjuvant

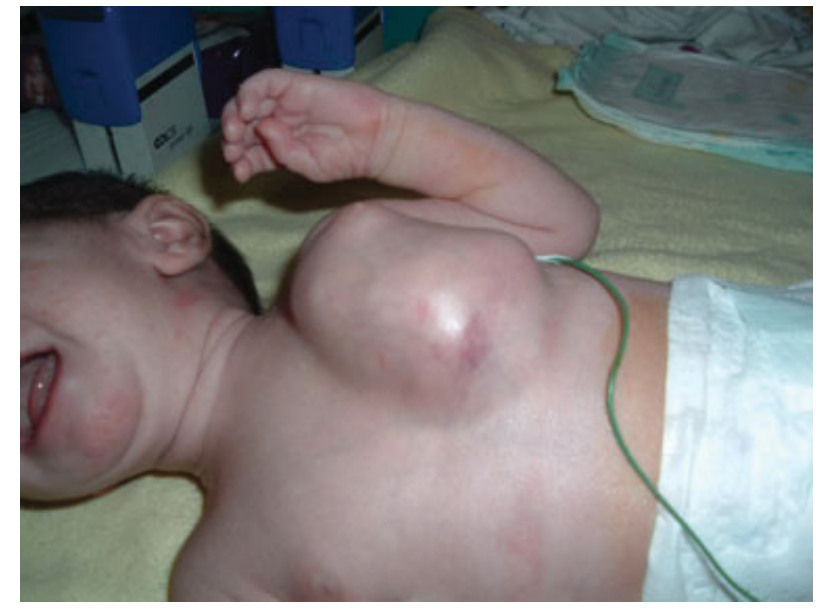

Fig. 4 Chest mass on the 16th day.

chemotherapy and to surgically remove the mass promptly, and planned to treat the remnants conservatively. The specimen was pear-shaped, about $10 \mathrm{~cm}$ long and $5 \mathrm{~cm}$ wide, and mostly defined with microscopic invasion to the intercostal muscles four to six and to the pectoralis muscles. The lesion was removed with about two-thirds of the pectoralis muscle to achieve a tumor-free border. Safety margin was approximately $2 \mathrm{~cm}$ at the pectoralis side; at the chest wall side the lesion was dissected from the wall and intercostal muscles, where microscopic lesions seen on frozen section were left in site. The skin was resected partially, so the defect could have been closed during the operation. At this point, the team that included the pediatric surgeon, the neonatologist and the pediatric oncologist, faced the dilemma of whether to proceed and resect a considerable part of the anterior chest wall, or to terminate the operation and rely on chemotherapy. Given that the final pathologic diagnosis was still pending and that a wide chest wall resection would have been mutilating, it was decided to end the operation, follow-up the infant, receive the final histopathologic results, administer chemotherapy, and consider additional surgery at a later date.

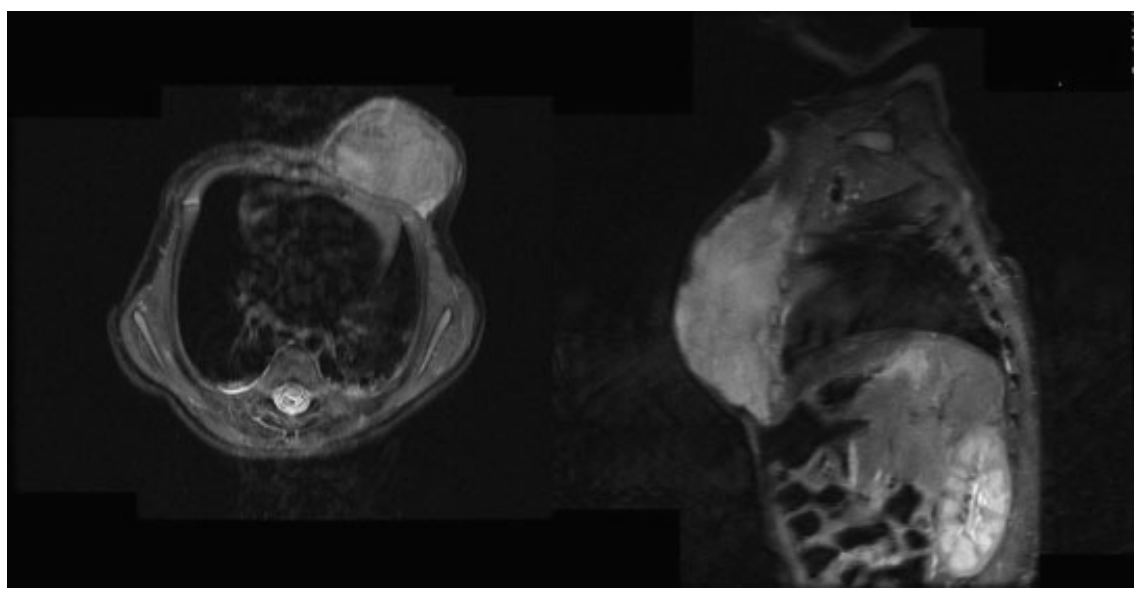

Fig. 3 MRI of the mass performed on the 13th day (right: sagittal view; left: transverse view). MRI, magnetic resonance imaging. 
On histopathologic examination, sections from the lesion showed skeletal muscle with extensive infiltration by a mass composed of sheets and bundles of spindle cells with focal coagulative necrosis accounting for about $10 \%$ of the area of the tumor. The tumor cells had round-to-oval nuclei with dark chromatin and indistinct eosinophilic cytoplasm with indistinct cell borders. There was a vaguely fascicular architecture in some areas and the tumor cells formed sheets in other areas. Mitoses and individual cell necrosis were present. The mitotic rate was approximately 12 per 10 high-power fields. The tumor cells showed focal strong nuclear reactivity for myogenin in areas away from the entrapped skeletal muscle and weak cytoplasmic reactivity for muscle-specific actin. As the tumor did not show any alveolar features, a rhabdomyosarcomata component with an embryonal subtype was a better diagnostic fit than fibrosarcoma.

The final histopathologic diagnosis was embryonal rhabdomyosarcoma. According to Intergroup Rhabdomyosarcoma Study group (IRSG) surgical-pathologic grouping system, the lesion was of group IIa, since it was a localized tumor, grossly removed with microscopic involved margins. Also, according to the IRSG staging system, it was of stage 3, as it was larger than $5 \mathrm{~cm}$, with no regional lymph node involvement and no metastases. $^{2}$

Chemotherapy included nine courses of ifosfamide + vincristine + actinomycin every 3 weeks, followed by maintenance with oral Cytoxan (Bristol-Myers Squibb Company, Princeton, New Jersey) and Vinorelbine (EBEWE Pharma, Unterach, Austria) for 6 months. Irradiation was omitted because of the patient's young age and the rarity of these tumors in this age.

MRI findings at the age of 21 months were normal. After 7 years of follow-up in the outpatient pediatric oncology clinic, the toddler is currently healthy and thriving, and there is no further evidence of neoplasm, including no disabilities and normal function of the upper extremities ( - Fig. 5).

\section{Discussion}

Only approximately 4,300 rhabdomyosarcoma cases have been described. ${ }^{2} \mathrm{~A}$ comprehensive PubMed search revealed

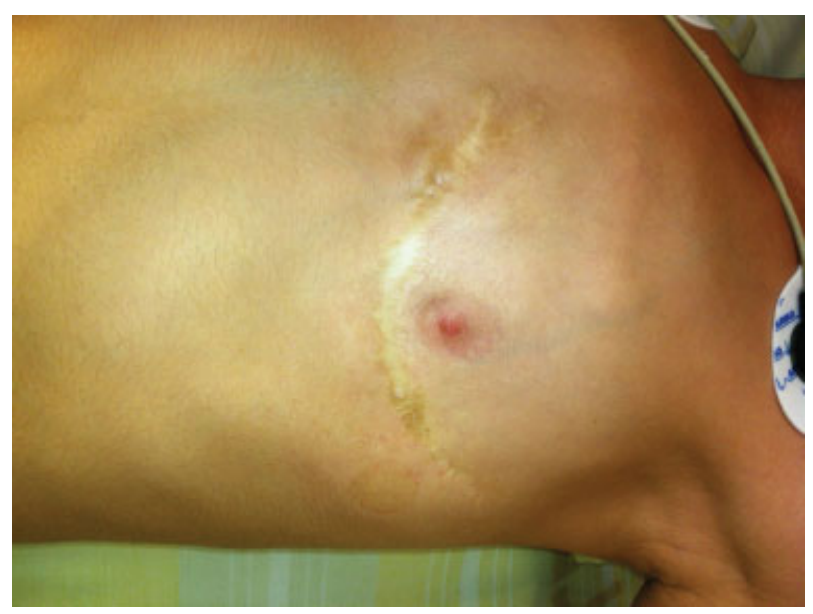

Fig. 5 Current picture of the child's chest. fewer than 40 reports of neonatal rhabdomyosarcoma, alone, or associated with other findings-which are about $1 \%$ of all cases of rhabdomyosarcoma cases. These reports are spread over approximately 40 years, during which classification and treatment recommendations were changed several times. Moreover, the reports were published by different disciplines in medicine, and, as such, reflect different viewpoints, so that it is difficult to compare the data they contain.

Current international classification of rhabdomyosarcoma is by histologic type: superior prognosis is designated for botryoid rhabdomyosarcoma and spindle cell rhabdomyosarcoma, intermediate prognosis is designated for embryonal rhabdomyosarcoma, and poor prognosis is designated for alveolar rhabdomyosarcoma and undifferentiated sarcoma, while the prognosis of the subtype rhabdomyosarcoma with rhabdoid features is not evaluable. ${ }^{3}$ A molecular classification of rhabdomyosarcoma, based on genomic analysis at the time of diagnosis, has been proposed. ${ }^{4}$ Postsurgical classification on a scale from 1 to 4 considers the resectability of the lesion, regional node involvement, and the presence of metastases.

Regarding the diagnosis of the current case, a different lesion, difficult to distinguish from rhabdomyosarcoma is infantile myofibromatosis, one of the most common benign fibrous tumors of infancy. Infant myofibromatosis is characterized by the presence of two different zones: the periphery of these lesions contains short fascicles of spindle-shaped cells, whereas the central portion consists of a hemangiopericytoma -like proliferation, interspersed with poorly differentiated polygonal or spindle-shaped cells. Necrosis and calcification are sometimes in the center of the lesion. Mild atypia and scattered generally few, mitotic figures can also be seen. Immunohistochemically, infantile myofibromatosis is reactive to actin but negative to desmin and myogenin. In contrast, the lesion presented in this case showed peripheral necrosis including individual cell necrosis and a high mitotic rate of 12 per 10 high power fields. In addition, it was positive for markers of muscular tissue (actin and desmin) and for a highly specific marker of skeletal muscle cells such as myogenin, including in areas away from the entrapped nontumoral muscle fibers. These features were also corroborated by an international expert on pediatric soft tissue tumor cells as noted above.

Therapy for rhabdomyosarcoma consists of a coordinated approach involving surgery, irradiation therapy, and chemotherapy. The IRSG conducted four studies on treatment of rhabdomyosarcoma, and their recommendations are the basis for the IRS-V treatment protocol ${ }^{2}$ as follows: it is preferable to try to remove all visible tumors, if this is feasible without extensive morbidity. The extent of disease at diagnosis affects prognosis. Re-excising an incompletely removed tumor is worthwhile if acceptable form and function can be preserved. The eye, vagina, and bladder can usually be saved. Irradiation is not necessary for children with localized, completely excised embryonal rhabdomyosarcoma, but it is indicated for all other groups.

In the current case, we macroscopically excised the tumor in its entirety. To save functionality, we decided not to resect a considerable part of the neonate's anterior chest wall; and due 
to the young age and the scarcity of this type of malignancy in this age, we decided not to irradiate. Rather, we chose to rely on chemotherapy alone to eradicate the microscopic intercostal remnants. Chemotherapy usually includes vincristine, actinomycin $\mathrm{D}$, and cyclophosphamide in combination with irradiation. Adding doxorubicin, cisplatin, etoposide, and ifosfamide was not shown to significantly improve survival of patients with gross residual or metastatic disease. ${ }^{2}$ Perhaps we should have discussed the case with the IRSG, despite the rarity of the neonatal type. Yet, the fast growing up rate of the lesion and the invasion to adjacent tissues led us to resect the tumor promptly, and conservatively treat the remnants.

In conclusion, neonatal rhabdomyosarcoma of the chest wall is a very rare entity. In this case, we described a lesion with a fast growing up rate. Almost 2 weeks passed while awaiting the final results of the pathologic and MRI studies; wishfully, it could be sooner. The efficient communication with surgical, oncologic, and pathologic consultants contributed to the fast decision-making and prompt surgery. Longterm follow-up revealed that the decisions taken were correct. Although, there is a common recommended protocol of treatment, sometimes one should adapt it to individual needs. Perhaps such a fast rate growing up lesion should be promptly removed, even before the establishment of chemotherapy.

\section{References}

1 Weiss SW, Goldeblum JR. Rhabdomyosarcoma. In: Weiss SW, Folpe AL, eds. Enzinger and Weiss's Soft Tissue Tumors. 4th ed. St. Louis, MO: CV Mosby; 2001:595-632

2 Raney RB, Maurer HM, Anderson JR, et al. The Intergroup Rhabdomyosarcoma Study Group (IRSG): major lessons from the IRS-I through IRS-IV studies as background for the current IRS-V treatment protocols. Sarcoma 2001;5(1):9-15

3 Newton WA Jr, Gehan EA, Webber BL, et al. Classification of rhabdomyosarcomas and related sarcomas. Pathologic aspects and proposal for a new classification-an Intergroup Rhabdomyosarcoma Study. Cancer 1995;76(6):1073-1085

4 Davicioni E, Anderson MJ, Finckenstein FG, et al. Molecular classification of rhabdomyosarcoma-genotypic and phenotypic determinants of diagnosis: a report from the Children's Oncology Group. Am J Pathol 2009;174(2):550-564 